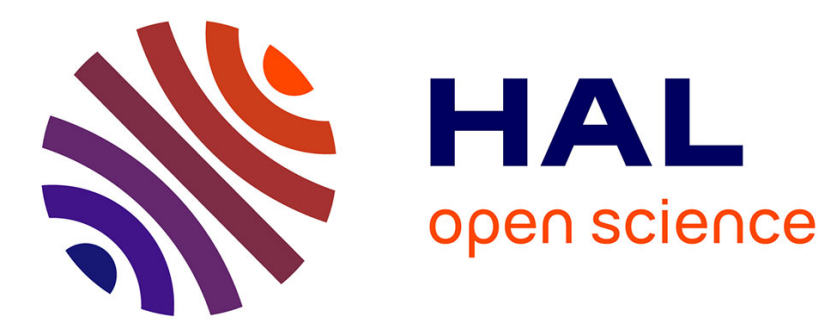

\title{
Mesure du travail de sortie du système BI/BI (0001)
}

J. Hölzl, C. Heimlich, J.P. Chauvineau, C. Pariset

\section{To cite this version:}

J. Hölzl, C. Heimlich, J.P. Chauvineau, C. Pariset. Mesure du travail de sortie du système BI/BI (0001). Revue de Physique Appliquée, 1984, 19 (5), pp.361-366. 10.1051/rphysap:01984001905036100 . jpa-00245204

\section{HAL Id: jpa-00245204 https://hal.science/jpa-00245204}

Submitted on 1 Jan 1984

HAL is a multi-disciplinary open access archive for the deposit and dissemination of scientific research documents, whether they are published or not. The documents may come from teaching and research institutions in France or abroad, or from public or private research centers.
L'archive ouverte pluridisciplinaire HAL, est destinée au dépôt et à la diffusion de documents scientifiques de niveau recherche, publiés ou non, émanant des établissements d'enseignement et de recherche français ou étrangers, des laboratoires publics ou privés. 


\title{
REVUE DE PHYSIQUE APPLIQUÉE
}

Revue Phys. Appl. 19 (1984) 361-366

MAI 1984, PAGE 361

Classification

Physics Abstracts

$73.30-68.55$

\section{Mesure du travail de sortie du système BI/BI (0001)}

\author{
J. Hölzl, C. Heimlich \\ Fachbereich Physik, Université de Kassel, \\ D-3500 Kassel, R.F.A.

\section{J. P. Chauvineau et C. Pariset (*)}

Institut d'Optique, Laboratoire associé au C.N.R.S., Centre Universitaire d'Orsay, Bât. 503, B.P. 43, 91406 Orsay Cedex, France

(Reçu le 28 septembre 1983, révisé le 11 janvier 1984, accepté le 13 février 1984)

\begin{abstract}
Résumé. - La mesure de la variation du travail de sortie $\Delta \Phi$ de couches minces de bismuth $(0001)$ (épaisseur $\approx 250 \AA$ ) en fonction du recouvrement $\theta$ en bismuth $(0<\theta \lesssim 2,5 \mathrm{MC})$ a été effectuée à différentes températures $T$ $(22 \leqslant T \leqslant 420 \mathrm{~K})$. A l'aide d'un modèle de Topping modifié, les résultats pour les faibles recouvrements $(\theta \lesssim 0,5 \mathrm{MC})$ nous permettent de déterminer le moment dipolaire effectif moyen $p_{\text {eff }}$ par adatome et d'estimer sa polarisabilité effective $\alpha$. Nous obtenons : $p_{\text {eff }}(22 \mathrm{~K})=(0,29 \pm 0,02) \mathrm{D}, p_{\text {eff }}(76 \mathrm{~K})=(0,14 \pm 0,02) \mathrm{D}$, $p_{\text {eff }}(100 \mathrm{~K})=(0,03 \pm 0,02) \mathrm{D}$ et $\alpha=(89 \pm 6) \AA^{3}$. Dans cette notation $p_{\text {eff }}(22 \mathrm{~K})$ correspond au moment dipolaire $p_{0}$ d'un adatome de bismuth isolé sur une terrasse $(0001)$ de bismuth. De plus, nous proposons une interprétation des courbes $\Delta \Phi(\theta)_{T}$ dans la gamme de recouvrement considérée à l'aide d'un modèle de croissance de la couche adsorbée dépendant de la température du substrat. Ce modèle est semblable à celui utilisé pour l'interprétation des variations de la résistance électrique de ce même système.
\end{abstract}

\begin{abstract}
Measurement of the work function variation $\Delta \Phi$ of bismuth thin films ( $250 \AA$ in thickness) as a function of bismuth surface coverage $\theta(0<\theta \lesssim 2.5 \mathrm{ML})$ was made at different temperatures $T(22 \leqslant T(\mathrm{~K}) \leqslant 420)$. Using a modified Topping model, the result for low coverages $(\theta \leqslant 0.5 \mathrm{ML})$ allow to determine the mean effective dipole moment $p_{\text {eff }}$ of an adatom and to estimate its effective polarizability $\alpha$. We obtain : $p_{\text {eff }}(22 K)=$ $(0.29 \pm 0.02) \mathrm{D} ; p_{\text {eff }}(76 \mathrm{~K})=(0.14 \pm 0.02) \mathrm{D} ; p_{\text {eff }}(100 \mathrm{~K})=(0.03 \pm 0.02) \mathrm{D} ;$ and $\alpha=(89 \pm 6) \AA^{3}$. In this notation, $p_{\text {eff }}(22 \mathrm{~K})$ corresponds to the dipole moment $p_{0}$ of an isolated bismuth adatom adsorbed on a (0001) terrace. Moreover, to account for the $\Delta \Phi(\theta)_{T}$ curves over the surface coverage range studied we propose to use a growth model depending on the substrate temperature for the adsorbed layer. This model is similar to that suitable for interpreting electrical resistivity changes previously measured on the same system.
\end{abstract}

\section{Introduction.}

L'influence de la rugosité à l'échelle atomique sur la réflexion des électrons de conduction par une surface a été discutée par Müser [1]. En appliquant la théorie de l'effet dimensionnel établie par Fuchs [2] et Sondheimer [3] à l'interprétation de la conductibilité

(*) Nouvelle adresse : Université de Clermont Ferrand II, Laboratoire de Physique des milieux condensés, Les Cézeaux, BP 45, 63170 Aubière, France. électrique de couches métalliques superposées, Lucas [4] a mis en évidence la variation du coefficient de réflexion spéculaire provoquée par la déformation de la surface libre d'une couche mince.

Chauvineau [5] et Pariset [6] ont mesuré les variations de la résistance électrique de couches minces de bismuth $(300 \AA<e<2000 \AA)$ en fonction du recouvrement $\theta$ en bismuth $(0<\theta \lesssim 6 \mathrm{MC})$ et de la température $T(15 \mathrm{~K}<T<420 \mathrm{~K})$. Ils ont développé un modèle permettant de relier leurs résultats à la diffusion superficielle des espèces adsorbées [7]. 
D'autre part, Smoluchowski [8] a montré que le travail de sortie dépend aussi de la variation de la rugosité superficielle. Hölzl et al. [9-11] ont mesuré les variations du travail de sortie en fonction du recouvrement $\theta$ et de la température $T$ pour le système $\mathrm{Ni} / \mathrm{Ni}(h k l)$. Ils en ont déduit les énergies d'activation pour les phénomènes de migration des adatomes, et les moments dipolaires pour les adatomes en fonction du nombre de premiers voisins.

Dans cette étude, nous avons mesuré les variations du travail de sortie et de la résistance électrique dans le cas du couple $\mathrm{Bi} / \mathrm{Bi}(0001)$. Nous montrons que les deux méthodes d'analyse donnent des résultats similaires en excellent accord avec le modèle de Chauvineau.

\section{Méthode expérimentale.}

La figure 1 montre le schéma de l'appareillage utilisé. Il est composé d'un porte-échantillon et d'un pendule décrit par Hölzl et al. [12] permettant la mesure en continu de la fonction travail de sortie. L'ensemble de cet appareillage est monté dans une enceinte ultravide où la pression est inférieure à $5 \times 10^{-8} \mathrm{~Pa}$.

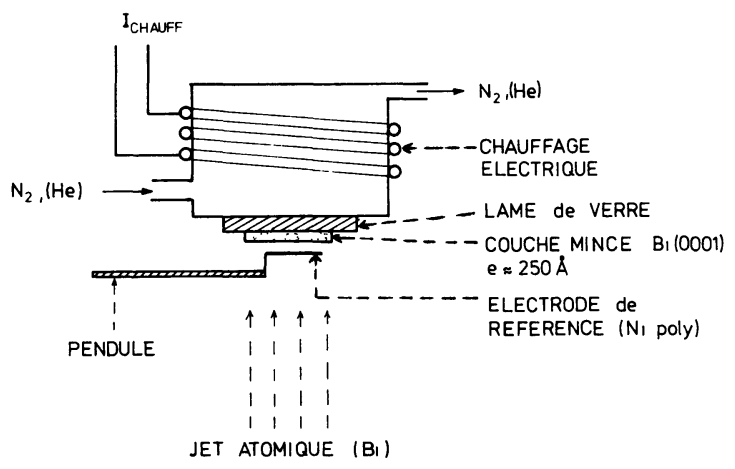

Fig. 1. - Schéma du système de mesure montrant le porteéchantillon (bloc de cuivre) avec réfrigération $\left(\mathrm{N}_{2}, \mathrm{He}\right.$ liquide) et chauffage électrique, le support de verre de l'électrode de bismuth déposée par évaporation thermique qui constitue le substrat pour la condensation de nouveaux atomes de bismuth (adsorbat), le pendule qui oscille perpendiculairement au plan du dessin, avec l'électrode de référence en nickel polycristallin.

[Experimental setup : Sample holder (copper block) cooled (liquid $\mathrm{N}_{2}$ or $\mathrm{He}$ ) or heated (electrical resistance); Bismuth electrode (thin film) used as a substrate for bismuth adatom condensation (adsorbate); Pendulum with reference electrode in polycrystalline nickel oscillating in a plan parallel to the substrate surface.]

Le porte-échantillon est constitué d'un bloc de cuivre dont la température peut varier de $20 \mathrm{~K}$ à $450 \mathrm{~K}$. La température est mesurée à l'aide d'un thermocouple $(\mathrm{Ni}-\mathrm{Cr} / \mathrm{Ni})$ avec une précision de $5 \mathrm{~K}$ à $T=20 \mathrm{~K}$ et de $0,5 \mathrm{~K}$ à température ambiante. Pendant une expérience, la stabilité de la température du substrat est de $\Delta T= \pm 0,5 \mathrm{~K}$.
Les couches minces de bismuth $(250 \AA<e<1100 \AA)$ sont déposées par évaporation de bismuth pur $(>99,999 \%$ ) à température ambiante sur des lames de verre fixées sur le bloc de cuivre. L'épaisseur $e$ des couches minces est mesurée à l'aide d'un quartz piézoélectrique avec une précision de $1 \%$. Après le dépôt du bismuth, le porte-échantillon est chauffé pendant une heure à $420 \mathrm{~K}$.

La conséquence de ce traitement thermique est une recristallisation de la couche mince de bismuth observée par Abdelmoula et al. [13] en microscopie électronique. La couche est composée de monocristaux d'un diamètre d'environ $2500 \AA$. Les monocristaux présentent à la surface de la couche mince des terrasses d'orientation (0001) de dimension latérale supérieure à $100 \AA$ qui sont séparées par des marches monoatomiques. La vitesse de dépôt est de 6 monocouches par minute pendant la croissance de la couche et de 3 à 4 monocouches par heure pendant la mesure du travail de sortie.

La couche mince de bismuth constitue l'une des électrodes de notre système de mesure. L'électrode de référence en nickel polycristallin est fixée à l'extrémité d'une tige d'acier. A l'autre extrémité de cette tige est fixée un morceau de fer doux. Celui-ci, placé dans un champ magnétique variable, peut faire osciller l'électrode de référence dans un plan parallèle à la surface de la couche mince à une distance d'environ $0,1 \mathrm{~mm}$ en dessous de celle-ci. La fréquence de cette oscillation est de $28 \mathrm{~Hz}$ et l'amplitude pic à pic est de $25 \mathrm{~mm}$ environ.

Le détail de la mesure du travail de sortie à l'aide de cet appareillage est décrit ailleurs [14].

\section{Résultats.}

La figure $2 a$ présente les résultats expérimentaux des mesures du travail de sortie de couches minces de bismuth $(e \approx 250 \AA)$ évaporées sur des lames de verre, sur lesquelles sont déposées des atomes de bismuth.

Les courbes représentent les changements du travail de sortie $\Delta \Phi(\mathrm{meV})$ en fonction du recouvrement $(\theta)$ en monocouches (MC) dans la gamme de $0<\theta \lesssim$ 2,5 MC pour différentes températures du substrat $(22 \mathrm{~K}<T<420 \mathrm{~K})$. La variation du travail de sortie est indépendante de l'épaisseur des couches minces, dont la valeur était comprise entre 250 et $1100 \AA$.

A la température $T=22 \mathrm{~K}$, le travail de sortie varie pour les très faibles recouvrements $(\theta \lesssim 0,05 \mathrm{MC})$ de façon presque linéaire avec une pente $\left.\frac{\mathrm{d} \Phi}{\mathrm{d} \theta}\right|_{\theta \rightarrow 0}=-1,05 \mathrm{eV} \cdot \mathrm{MC}^{-1}$. Lorsque le recouvrement augmente $(\theta>0,05 \mathrm{MC})$ la courbe n'est plus linéaire et atteint un minimum de $\Delta \Phi=-(70 \pm 2) \mathrm{meV}$ à $\theta \approx 0,2 \mathrm{MC}$. La remontée de la courbe après le minimum est importante dans la gamme de $0,2 \mathrm{MC}<\theta \lesssim 0,8 \mathrm{MC}$ et elle approche une valeur de saturation pour $\theta>0,8 \mathrm{MC}$.

L'allure de la courbe à $T=76 \mathrm{~K}$ est similaire, 

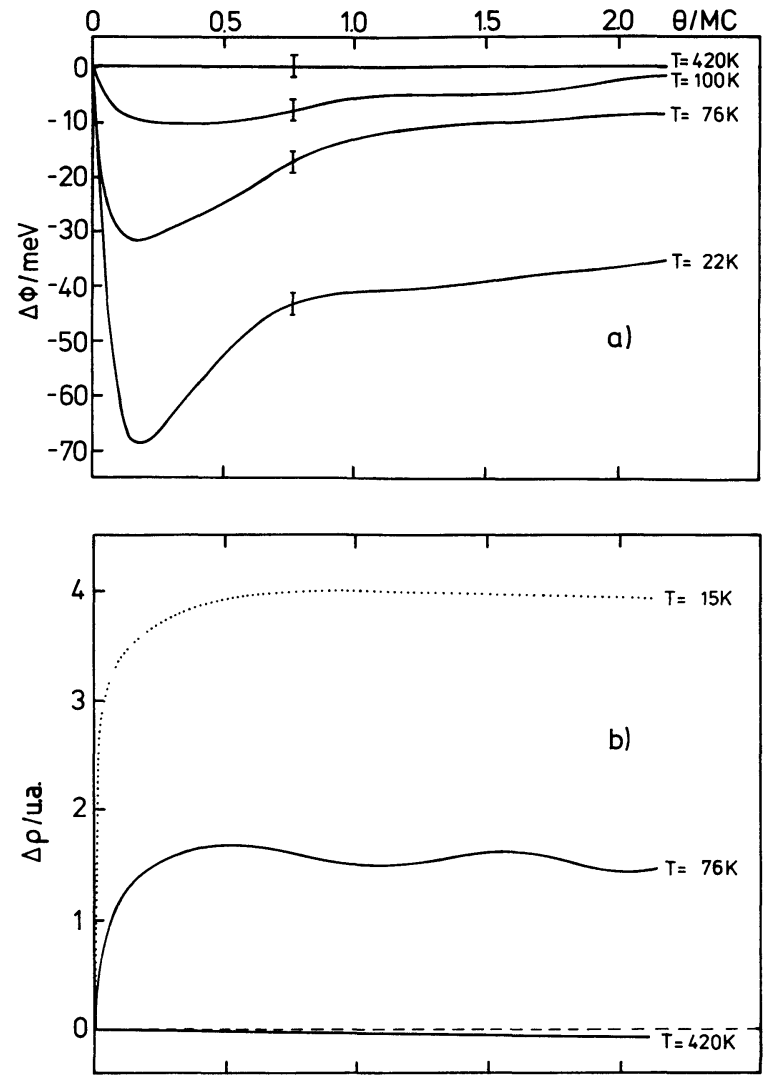

Fig. 2. - a) Variation du travail de sortie $\Delta \Phi(\mathrm{meV})$; b) Variation de la résistivité électrique $\Delta \rho$ (u.a.) en fonction du recouvrement $\theta(\mathrm{MC})$ à différentes températures du substrat $T(15 \mathrm{~K}<T<420 \mathrm{~K})$. La courbe pointillée est extraite de la référence [15].

[a) Work function variation $\Delta \Phi(\mathrm{meV})$; b) Electrical resistivity variation $\Delta \rho$ (a.u.) as a function of surface coverage $\theta(\mathrm{ML})$ for different substrate temperatures $T(15 \mathrm{~K}<T<$ $420 \mathrm{~K})$. The dotted curve is taken from reference [15].]

mais la valeur du minimum $\Delta \Phi=-(30 \pm 2) \mathrm{meV}$ est inférieure.

La courbe à $T=100 \mathrm{~K}$ est qualitativement la même qu'à $22 \mathrm{~K}$.

Pour cette température la variation maximale du travail de sortie est $\Delta \Phi=-(10 \pm 2) \mathrm{meV}$. Dans ce cas, on ne peut pas déterminer avec précision la position du minimum.

Pour les hautes températures $(T=420 \mathrm{~K})$, le travail de sortie ne varie plus en fonction du nombre d'atomes adsorbés.

$\mathrm{La}$ figure $2 \mathrm{~b}$ présente les variations de la résistance électrique $\Delta \rho$ (u.a.) des couches minces de bismuth en fonction du recouvrement $\theta$ (MC) en bismuth pour trois températures différentes $(15 \mathrm{~K}, 76 \mathrm{~K}$ et $420 \mathrm{~K})$.

Pour pouvoir comparer directement nos résultats, les mesures ont été faites dans les mêmes conditions expérimentales.

Les résultats de nos mesures de variation de la résistance électrique sont en très bon accord avec ceux de Pariset et al. [15]. La courbe en pointillés $(T=15 \mathrm{~K})$ de la figure $2 b$ est extraite de cette référence.

\section{Discussion.}

Une surface métallique sur laquelle on a déposé $N_{v}$ adatomes par $\mathrm{cm}^{2}$ (moment dipolaire $p_{v}$ ) présente une variation $\Delta \Phi$ de son travail de sortie $\Phi$ par rapport à la surface propre. Etant donnée l'influence des premiers voisins d'un adatome $(v=0,1, \ldots, 6$ pour le réseau superficiel hexagonal), on peut décrire $\Delta \Phi$, pour les systèmes homogènes et hétérogènes, à l'aide d'une équation de Helmholtz modifiée :

$$
\Delta \Phi=-C \sum_{v=0}^{6} N_{v} p_{v} .
$$

Dans cette équation, $C$ est égal à $300.4 \pi e_{0} \times 10^{-18} \mathrm{~V}$ $\mathrm{cm}^{2} . \mathrm{D}^{-1}$ avec la charge élémentaire $e_{0}$, pour des moments dipolaires en Debye (D).

Dans notre expérience, des atomes de bismuth sont condensés sur une surface (0001) de bismuth. Généralement ces atomes sont mobiles sur la surface si la température du substrat n'est pas trop basse et si leur énergie thermique résiduelle propre est suffisante. Dans la suite nous observons la dépendance $\Delta \Phi=f(\theta)$ pour différentes températures constantes pendant une expérience. Nous nous intéresserons d'abord au cas particulier pour lequel le recouvrement est très faible et les atomes immobiles (adatomes isolés sans interaction latérale). Puis un cas plus général (adatomes avec interaction latérale) sera discuté dans un deuxième paragraphe.

4.1 Adatomes Sans interaction latérale. - A la température $T=22 \mathrm{~K}$, les atomes de bismuth condensés sur une surface (0001) de bismuth sont fixés sur des sites d'adsorption distribués au hasard. Dans le cas des très faibles recouvrements $(\theta \lesssim 0,05 \mathrm{MC})$ l'interaction des adatomes n'existe pratiquement pas (adatomes isolés). La variation du travail de sortie est donc directement proportionnelle au nombre de dipôles de moment dipolaire $p_{0}$. On peut alors calculer à l'aide de l'équation (1) le moment dipolaire moyen des atomes de bismuth à partir de la pente de la partie linéaire de la courbe pour $T=22 \mathrm{~K}$ :

$$
p_{0}=-\left.\frac{1}{C N_{0}} \frac{\mathrm{d} \Phi}{\mathrm{d} \theta}\right|_{\theta \rightarrow 0} .
$$

Dans cette équation $\theta . N_{0}$ est le nombre $N$ des atomes adsorbés par $\mathrm{cm}^{2}: N_{0}$ représente le nombre de sites d'adsorption par $\mathrm{cm}^{2}$ et $\theta$ le recouvrement en monocouches (MC). La valeur de $p_{0}$ ainsi calculée est : $p_{0}=(0,25 \pm 0,1) \mathrm{D}$.

Toutefois on doit considérer sur les terrasses d'orientation (0001) les deux sites d'adsorption différents qui se distinguent par le nombre de seconds voisins du substrat. Ces deux sites sont en effet occupés uniformément parce que les adatomes sont distribués au hasard. Pour cette raison, notre valeur moyenne 
de $p_{0}$ est composée de deux contributions $p_{0}^{\prime}$ et $p_{0}^{\prime \prime}$ de poids presque similaire $\left({ }^{1}\right)$ dans $p_{0}\left(p_{0}=\frac{1}{2}\left(p_{0}^{\prime}+p_{0}^{\prime \prime}\right)\right)$.

L'incertitude relativement grande sur le moment dipolaire $p_{0}(\Delta p \approx 50 \%)$ est la conséquence de la faible variation du travail de sortie et de la prise en compte d'une petite gamme du recouvrement $(0<\theta \lesssim 0,05 \mathrm{MC})$ pour l'évaluation de $p_{0}$.

Dans le paragraphe suivant $p_{0}$ sera calculé à partir d'une plus grande gamme de recouvrements $(0<\theta \lesssim$ $0,5 \mathrm{MC}$ ) ce qui aura pour effet de diminuer l'incertitude.

4.2 Adatomes aVeC interaction latérale. - Pour les recouvrements supérieurs à $0,05 \mathrm{MC}$, on doit tenir compte de l'interaction des adatomes car ils forment, même à $T=22 \mathrm{~K}$, des amas sur les terrasses. Pour des températures plus hautes $(T>45 \mathrm{~K})$ [5], les processus de migration des adatomes entraînent la formation de multimères (dimères, trimères, etc.) même pour les faibles recouvrements.

Lorsque ces multimères sont composés d'adatomes qui étaient adsorbés isolément au cours du dépôt (moment dipolaire $p_{0}$ ), les dipôles formant les multimères se dépolarisent mutuellement. Dans le cas du réseau hexagonal, les atomes dans les amas ont les moments dipolaires $p_{1}, \ldots, p_{6}$ si on considère seulement l'influence des premiers voisins. Dans notre discussion nous nous intéresserons à la valeur du moment dipolaire moyen $p_{\text {eff }}$ d'un adatome

$$
p_{\text {eff }}=\frac{1}{N} \sum_{v=0}^{6} p_{v} N_{v} .
$$

A l'aide d'un modèle de Topping modifié par Hölzl et al. [10], on peut calculer la variation du travail de sortie $\Delta \Phi$ en fonction du recouvrement $\theta$ pour une certaine température $T$ :

$$
\Delta \Phi(\theta, T)=-C \frac{p_{\mathrm{eff}}(T) \theta N_{0}}{1+\kappa(T) \alpha(T) \theta^{3 / 2} N_{0}^{3 / 2}} .
$$

Dans cette équation le facteur $\kappa(T)$ tient compte de la structure géométrique des adatomes dans les amas en fonction de la température, et $\alpha(T)$ est la polarisabilité effective des atomes de bismuth à la surface (0001) du substrat de bismuth.

La transformation de l'équation (4) donne une équation linéaire :

$$
-\frac{\theta}{\Delta \Phi}=\frac{1}{C \cdot N_{0} p_{\text {eff }}(T)}\left(1+\kappa(T) \alpha(T) N_{0}^{3 / 2} \cdot \theta^{3 / 2}\right) .
$$

Si on représente les valeurs expérimentales pour une

( ${ }^{1}$ ) En effet la probabilité d'occupation des deux types de sites n'est pas la même, car les énergies d'adsorption sont légèrement différentes [5]. certaine température sous la forme $\frac{\theta}{\Delta \Phi} v s . \theta^{3 / 2}$, on obtient une droite dans la gamme de recouvrements $0<\theta \lesssim 0,4 \mathrm{MC}$ (Fig. 3). Cela justifie l'application du modèle de Topping modifié pour ces recouvrements.

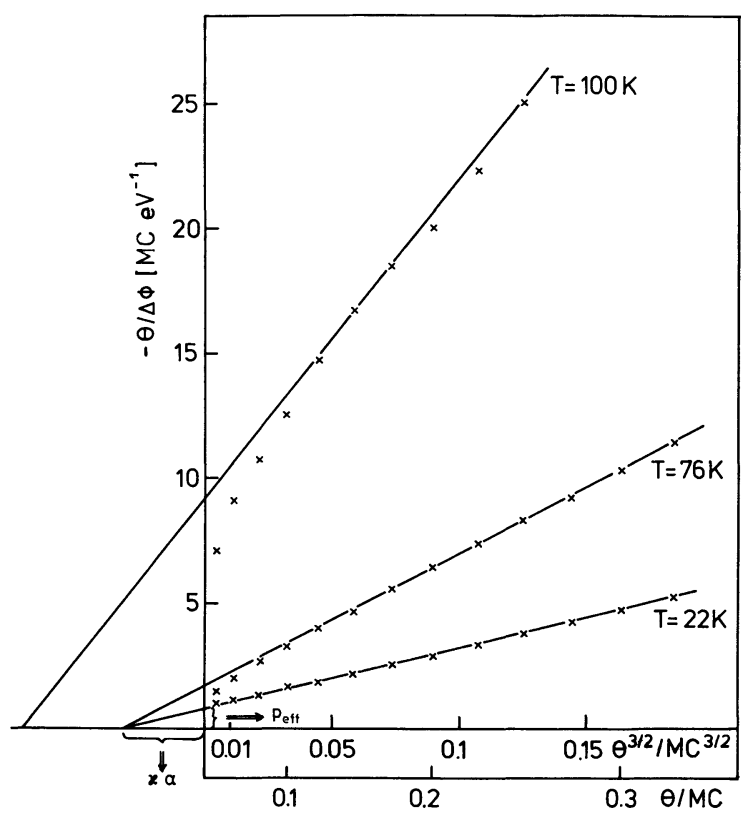

Fig. 3. - Variation de $\theta / \Delta \Phi\left(\mathrm{MC} \mathrm{eV}^{-1}\right) v s$. $\theta^{3 / 2}\left(\mathrm{MC}^{3 / 2}\right)$ à différentes températures $T(22 \leqslant T \leqslant 420(\mathrm{~K}))$. Les croix $(x)$ représentent les valeurs expérimentales extraites de la figure $2 \mathrm{a}$ et les lignes représentent les droites calculées. Les coordonnées à l'origine de ces droites permettent de calculer $p_{\text {eff }}$ et $\kappa . \alpha$. Les valeurs de $\theta(\mathrm{MC})$ sont également indiquées en abscisse.

[Variation of $\theta / \Delta \Phi\left(\mathrm{ML} \mathrm{eV}^{-1}\right)$ as a function of $\theta^{3 / 2}\left(\mathrm{ML}^{3 / 2}\right)$ at different temperatures $T(22 \mathrm{~K}<T<420 \mathrm{~K})$. The crosses $(x)$ represent the experimental values taken from figure $2 \mathrm{a}$ and the straight lines are calculated curves. The intercept of these lines with the coordinate axes allow to calculate the values of $p_{\text {eff }}$ and $\kappa . \alpha$. The values of $\theta(\mathrm{ML})$ are also indicated on the $X$-axis.]

Les points d'intersection des axes de coordonnées avec la droite obtenue par régression linéaire permettent de calculer les valeurs de $p_{\text {eff }}$ et de $\kappa . \alpha$. Le tableau I regroupe ces valeurs pour les différentes températures.

Tableau I.

\begin{tabular}{|c|c|c|}
\hline$T(\mathrm{~K})$ & $p_{\text {eff }}(\mathrm{D})$ & $\kappa \cdot \alpha\left(\AA^{3}\right)$ \\
\hline 22 & $0,29 \pm 0,02$ & $794 \pm 20$ \\
76 & $0,14 \pm 0,02$ & $812 \pm 20$ \\
100 & $0,03 \pm 0,02$ & $364 \pm 20$ \\
\hline
\end{tabular}

Pour contrôler le domaine de validité des constantes $\left(p_{\text {eff }}, \kappa \cdot \alpha\right)$, nous avons calculé $\Delta \Phi=f(\theta)$ à 
l'aide de ces valeurs jusqu'à un recouvrement de $0,8 \mathrm{MC}$ et nous avons comparé la valeur de $\Delta \Phi$ calculée avec celle mesurée (Fig. 4). Les valeurs mesurées (courbe pleine) sont en bon accord avec celles calculées (cercles) dans la gamme de recouvrement $0<\theta \lesssim 0,5 \mathrm{MC}$. La divergence entre la courbe et les valeurs calculées pour $T=22 \mathrm{~K}$ et $\theta>0,5 \mathrm{MC}$ est due à la croissance tridimensionnelle des amas sur les terrasses, qui n'est pas prise en compte par le modèle de Topping.

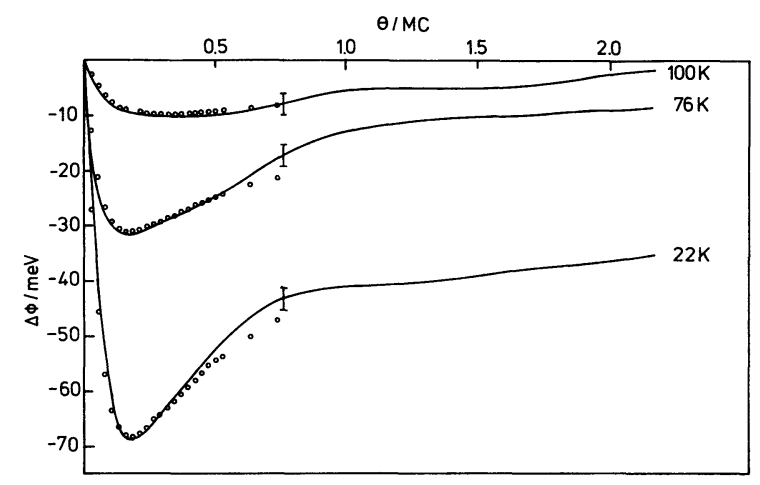

Fig. 4. - Comparaison des valeurs de $\Delta \Phi(\theta)$ mesurées (courbes en trait continu) avec les valeurs de $\Delta \Phi(\theta)$ calculées à l'aide de l'équation (4) avec les paramètres du tableau I (cercles).

[Comparison between the values of $\Delta \Phi(\theta)$ measured (curves) and calculated from equation (4) using the parameters of table I (circles).]

Les valeurs des moments dipolaires $p_{0}(\S 4.1)$ et $p_{\text {eff }}(22 \mathrm{~K})$ sont similaires aux incertitudes près. L'exploitation de la courbe à l'aide du modèle de Topping donne une valeur du moment dipolaire d'un adatome de bismuth isolé sur une terrasse (0001) de bismuth avec plus de précision que celle obtenue avec le modèle primitif de Helmholtz. Le modèle de Topping nous donne aussi la valeur de $\kappa \cdot \alpha=(794 \pm 20) \AA^{3}$, pour $T=22 \mathrm{~K}$ (Tableau I). Puisque les adatomes sont immobiles à cette température, on peut utiliser le facteur géométrique du réseau hexagonal : $\kappa=8,89$ suivant Topping. La polarisabilité effective $\alpha$ d'un adatome de bismuth sur une surface (0001) de bismuth est alors $\alpha=(89 \pm 6) \AA^{3}$. A l'aide de ces deux valeurs, $p_{\text {eff }}(22 \mathrm{~K})$ et $\alpha$, on peut calculer le moment dipolaire $p_{1}$ d'un adatome avec son voisin (adatome dans un dimère). Si on suppose que la loi permettant le calcul du champ dipolaire est applicable, même si on considère l'interaction des adatomes avec le substrat, alors on peut écrire :

$$
p_{1}=p_{0}\left(1-\frac{\alpha}{r^{3}}\right)
$$

soit, $p_{1}=(0,07 \pm 0,01) \mathrm{D}$.

La valeur de $p_{1}$ calculée à l'aide du modèle de Albano et al. [16] est $\approx 0,06 \mathrm{D}$.

Quand la température dépasse $40 \mathrm{~K}$, la mobilité des atomes adsorbés permet la formation de multimères sur les terrasses (0001) et la fixation d'atomes le long des marches de la surface initiale. Nous supposons que le nombre d'amas bidimensionnels diminue et que leur taille moyenne augmente lorsque respectivement la température et le taux de recouvrement augmentent. Dans ces mêmes conditions, le nombre moyen de premiers voisins $v$ par atome adsorbé augmente également; compte tenu de l'effet de dépolarisation, le moment dipolaire effectif défini par la relation (3) doit donc diminuer. L'existence de parties linéaires dans un domaine limité de recouvrement observé sur les courbes expérimentales qui représentent $(-\theta / \Delta \Phi)$ en fonction de $\theta^{3 / 2}$ (Fig. 3) peut provenir de la prépondérance dans cet intervalle de l'une des formes d'atomes adsorbés ayant un nombre de voisins $v$ fixé, ce qui entraîne, d'après la relation(3) : $p_{\text {eff }} \cong p_{v}$.

Les valeurs de $p_{\text {eff }}$ déterminées à 76 et $100 \mathrm{~K}$ à partir des droites de la figure 3 entre 0,1 et $0,3 \mathrm{MC}$ environ sont indiquées sur le tableau I; la comparaison avec la valeur obtenue à $22 \mathrm{~K}$ montre que $p_{\text {eff }}(T)$ diminue lorsque la température augmente. D'autre part, en dessous de 0,1 MC l'écart des points expérimentaux par rapport aux droites tracées sur la figure 3 à 76 et $100 \mathrm{~K}$ correspond bien à un accroissement de $p_{\text {eff }}$ lorsque le recouvrement $\theta$ diminue.

4.3 Comparaison DES VARIATIONS DU TRAVAIL DE SORTIE ET DE LA RÉSISTIVITÉ ÉLECTRIQUE. — Le dépôt d'atomes sur une couche mince métallique (épaisseur $e$ inférieure au libre parcours moyen des électrons de conduction dans le métal massif) a une influence significative sur la rugosité superficielle de cette couche et donc sur sa résistance électrique. La réflexion des électrons de conduction à la surface devient diffuse si la rugosité microscopique de la surface augmente et par conséquent la résistance électrique de la couche mince croît.

Le modèle de la croissance d'une couche adsorbée à différentes températures dans le cas $\mathrm{Bi} / \mathrm{Bi}(0001)$ développé par Chauvineau [7] à partir des mesures des variations de la résistance électrique $\Delta \rho$ en fonction du recouvrement est presque identique à celui que nous avons utilisé pour l'interprétation des résultats en $\Delta \Phi(\$ 4.1 .4 .2)$.

Dans les deux cas, la variation de la quantité considérée $(\rho$ ou $\Phi)$ à $T=15$ ou $22 \mathrm{~K}$ est plus grande que les variations observées à $T \geqslant 76 \mathrm{~K}$ (Fig. 2). Cet effet est dû à la diminution de la mobilité des adatomes lorsque la température décroît. A 15 ou $22 \mathrm{~K}$ la condensation aléatoire et l'absence de migration superficielle des adatomes $\mathrm{Bi} / \mathrm{Bi}(0001)$, à l'échelle de temps d'une expérience, ont pour conséquence l'apparition d'une forte rugosité à l'échelle atomique pendant la croissance de la couche même. Au-dessus de $40 \mathrm{~K}$, les adatomes peuvent migrer sur la surface (0001); ils se fixent le long des marches ou forment des amas de différentes tailles, ce qui a pour effet de 
réduire la rugosité de la surface par rapport au cas précédent $(T<40 \mathrm{~K})$.

A température égale ou supérieure à $76 \mathrm{~K}$, la taille moyenne des îlots commence par augmenter en même temps que le taux de recouvrement $\theta$. Finalement, à $\theta \approx 1 \mathrm{MC}$, la surface originale tend à se reproduire sous l'effet de la coalescence des îlots. Mais la nouvelle surface n'est pas identique à la surface initiale lisse. A cause d'une croissance tridimensionnelle, de petits amas apparaissent aussi sur les îlots qui forment la première monocouche et par conséquent celle-ci reste rugueuse. Une conséquence directe du mode de croissance de la couche adsorbée (monocouche par monocouche) est une variation périodique de la rugosité superficielle qui doit présenter un minimum chaque fois que l'on a déposé l'équivalent d'un nombre entier de monocouches. Cet effet devrait provoquer une modulation (avec une période d'une monocouche) des courbes de résistance électrique et du travail de sortie, si les températures choisies ne sont pas trop basses. En réalité seule la courbe $\Delta \rho(\theta)$ à $T=76 \mathrm{~K}$ montre une telle modulation dont l'amplitude reste cependant relativement faible par rapport à l'accroissement moyen de la résistivité.

Les variations majeures du travail de sortie d'une surface cristalline sont dues aux moments dipolaires de monomères et de dimères présents sur cette surface. Les moments dipolaires des adatomes situés au bord de marche des îlots contribuent très peu à la variation totale du travail de sortie à cause de la forte dépolarisation de ces dipôles. Lorsque le recouvrement augmente de $\theta=0$ à $\theta \approx 1 \mathrm{MC}$, une forte variation du travail de sortie est donc prévisible avec un minimum pour les faibles recouvrements $(\theta \approx 0,2 \mathrm{MC})$. Puis dans le cas idéal (absence de croissance tridimensionnelle) $\Delta \Phi$ devrait tendre vers 0 lorsque $\theta$ tend vers $\approx 1 \mathrm{MC}$. Pour un recouvrement supérieur à $\approx 1 \mathrm{MC}$ ce processus devrait se répéter.

Dans l'expérience réelle, nous devons considérer une croissance tridimensionnelle due à la formation d'amas sur la première monocouche encore incomplète. Nous observons donc un écart par rapport au travail de sortie initial $(\Delta \Phi \approx 15 \mathrm{meV})$ même pour $\theta \approx 1 \mathrm{MC}$. Si nous augmentons maintenant le recouvrement $(\theta \gtrsim 1 \mathrm{MC})$, les adatomes déposés se fixent aux multimères présents à la surface parce qu'ils ont la possibilité de migrer. Ils sont alors dépolarisés par les atomes formant les multimères. Cet effet de la dépolarisation des adatomes nouvellement déposés empêche une nouvelle diminution du travail de sortie. Nous observons au contraire une petite augmentation du travail de sortie qui s'explique par l'interaction des atomes des multimères et des nouveaux adatomes (dépolarisation réciproque).

Enfin, lorsque la température atteint $420 \mathrm{~K}$, la surface n'est pratiquement plus modifiée par les atomes condensés qui ont une mobilité suffisante pour venir se fixer sur des sites tels que les crans des marches de la surface initiale, ce qui entraîne la croissance de la couche sans déformation de sa surface libre. Le travail de sortie ne varie plus, et la faible décroissance de la résistivité électrique est due à l'augmentation d'épaisseur moyenne de la couche qui diminue l'importance relative de la diffusion des électrons de conduction par les surfaces.

\section{Remerciements.}

Ce travail a bénéficié d'un soutien financier du C.N.R.S. dans le cadre de l'A.T.P. internationale 1981.

\section{Bibliographie}

[1] Müser, H. A., Philos. Mag. 45 (1954) 1237.

[2] Fuchs, R., Proc. Cambridge Phil. Soc. 34 (1938) 100.

[3] Sondheimer, E. H., Advan. Phys. 1 (1952) 1.

[4] LuCas, M. S. P., Appl. Phys. Lett. 4 (1964) 73.

[5] Chauvineau, J. P., Surf. Sci. 93 (1980) 471.

[6] Pariset, C., Thèse Univers., Paris (1976).

[7] Chauvineau, J. P., J. Crystal Growth 53 (1981) 505.

[8] Smoluchowski, R., Phys. Rev. 60 (1941) 661.

[9] Hölzl, J., PorsCh, G., Thin Solid Films 28 (1975) 93.

[10] Hölzl, J., Porsch, G., Schrammen, P., Surf. Sci. 97 (1980) 529
[11] Schrammen, P., Hölzl, J., Surf. Sci. 130 (1983) 203

[12] Hölzl, J., Schrammen, P., Appl. Phys. 3 (1974) 353.

[13] Abdelmoula, K., Pardo, B., Pariset, C., Renard, D., Thin Solid Films 62 (1979) 273.

[14] Heimlich, C., Diplomarbeit Gesamthochschule Kassel (1983).

[15] Pariset, C., Vatamanyuk, V. I., J. Physique Lett. 39 (1980) L-283.

[16] Albano, E. V., Heras, J. M., Schrammen, P., Mann, M., HölzL, J., Surf. Sci. 129 (1983) 137. 